\title{
Low-Grade Fibromyxoid Sarcoma in the Left Gluteal Region Presenting as Sciatic Nerve Pain
}

\author{
Kazuhiko Hashimoto*, Kensuke Toriumi, Yukiko Hara, Shunki Iemura, Shunji Nishimura, \\ Masao Akagi
}

Department of Orthopedic Surgery, Kindai University Hospital, Osaka-Sayama City, Japan

Email: ^hazzhiko@med.kindai.ac.jp

How to cite this paper: Hashimoto, K., Toriumi, K., Hara, Y., Iemura, S., Nishimura, S. and Akagi, M. (2018) Low-Grade Fibromyxoid Sarcoma in the Left Gluteal Region Presenting as Sciatic Nerve Pain. Open Journal of Orthopedics, 8, 168-174. https://doi.org/10.4236/ojo.2018.84019

Received: March 20, 2018

Accepted: April 20, 2018

Published: April 23, 2018

Copyright ( $\odot 2018$ by authors and Scientific Research Publishing Inc. This work is licensed under the Creative Commons Attribution International License (CC BY 4.0).

http://creativecommons.org/licenses/by/4.0/

\begin{abstract}
Low-grade fibromyxoid sarcoma is a slowly growing soft tissue neoplasm, mostly affecting young individuals. It usually arises in a deep soft tissue of the lower limbs and trunk, but few cases of low-grade fibromyxoid sarcoma that presents sciatic nerve pain have been reported. We report a 34-year-old woman with a low-grade fibromyxoid sarcoma originating in the left gluteal region that initially presented as sciatic nerve pain; she had experienced this type of pain for 5 years before visiting our hospital. Magnetic resonance imaging revealed a tumor in the left gluteal region. After needle biopsy revealed it to be a low-grade fibromyxoid sarcoma, we performed the wide resection. Intra-operative findings revealed no tumor invasion into the sciatic nerve. No recurrence or metastasis has been detected 6 months post-surgery. Oncologists who encounter patients with sciatic nerve pain should consider the possibility of less common causes such as the low-grade fibromyxoid sarcoma found in our patient.
\end{abstract}

\section{Keywords}

Fibromyxoid Sarcoma, Sciatic Nerve, Obscure Tumors

\section{Introduction}

Low-grade fibromyxoid sarcoma is a rare malignancy that is often metastatic and typically develops in the deep soft tissue of the trunk or proximal extremities of young adults; it was first reported as a distinct entity by Harry Evans in 1987 [1]. Macroscopically, low-grade fibromyxoid sarcoma is a well-circumscribed mass. The excised surfaces of the tumors exhibit a fibrous, yellow-white appearance with glistening areas accompanied by the accumulation of a myxoid substance [1]. To our knowledge, low-grade fibromyxoid sarcoma that presents as 
sciatic nerve pain has not previously been reported. Herein, we report a low-grade fibromyxoid sarcoma of the left gluteal region presenting as sciatic nerve pain.

\section{Case Presentation}

A 34-year-old woman had been aware of a mass in her left gluteal region for the previous 5 years. She visited a nearby clinic because of sciatic nerve pain, whereupon she was referred to our facility. She had no remarkable medical history other than pain in her left leg. A $12 \times 13 \mathrm{~cm}$ elastic-hard mass was observed in her left gluteal region (Figure 1(a)). The mass was tender, and mobility between it and the skin was poor as was mobility within the mass itself. Her left leg pain, which was consistent with sciatic nerve pain, became severe upon compression of the mass. T1-weighted magnetic resonance imaging (MRI) showed a uniform low-intensity mass in the gluteal region (Figure 1(b) and Figure 1(c)), while T2-weighted MRI showed a mixed low- and high-intensity image (Figure 2(a) and Figure 2(b)). Accumulation was observed on 18F-fluorodeoxyglucose positron emission tomography (18FDG-PET) (Figure 2(c) and Figure 2(d)). The maximum standardized uptake value (SUV max) of the mass was 3.55; no accumulation was observed elsewhere in her body. A needle biopsy of her mass showed increased fibrous cells on a myxomatous background (Figure 3(a)). The nuclei of the cells were ovoid and spindle-shaped, and no necrotic regions or nuclear fission was observed. We considered myxoma or low-grade fibromyxoid sarcoma in the differential diagnosis; because a malignancy could not be ruled out, we performed wide resection (Figure $3(\mathrm{~b})$ and Figure $3(\mathrm{c})$ ). The sciatic nerve was located under the tumor separate from the gluteal muscle layer (Figure 3(b) and Figure 3(c)). Macroscopically, the resected specimen was elastic-hard and its margin was negative (Figure 3(d)). The histology of the resected specimen showed increased spindle cells on the myxomatous background and

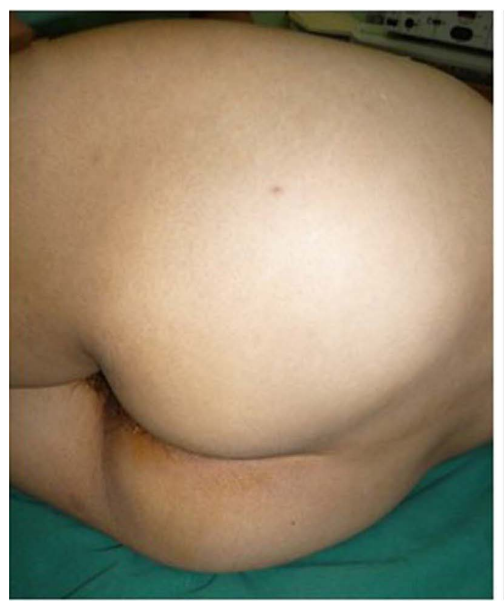

(a)

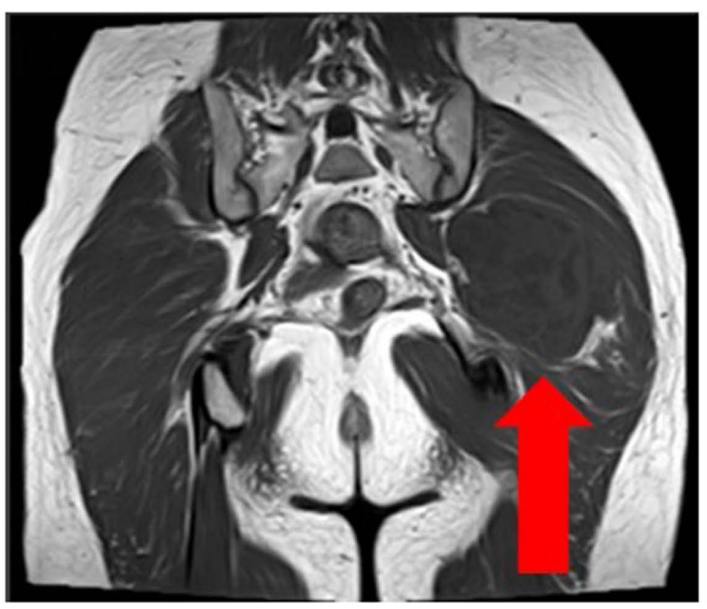

(b)

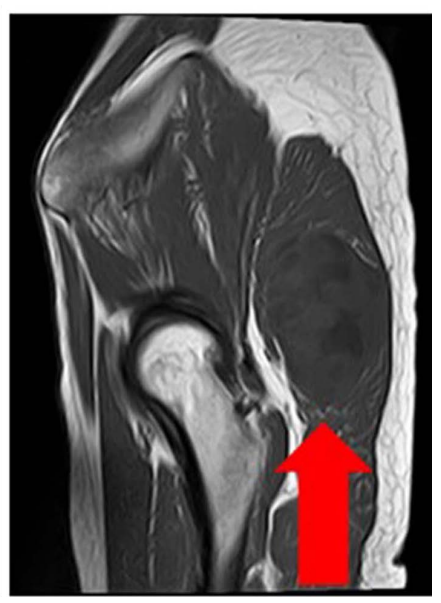

(c)

Figure 1. (a) Appearance of the upper-left side of the buttock on clinical examination; (b) T1-weighted coronal image of the tumor; (c) T1-weighted sagittal image of the tumor. Red arrows indicate the tumor mass. 


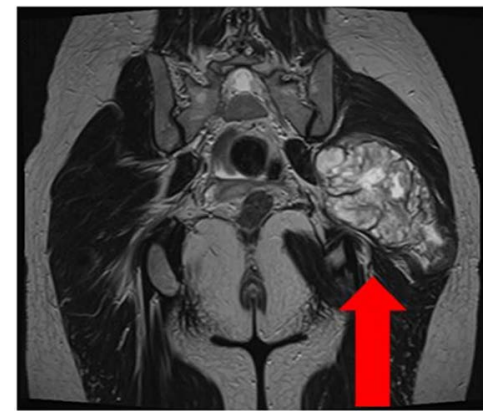

(a)

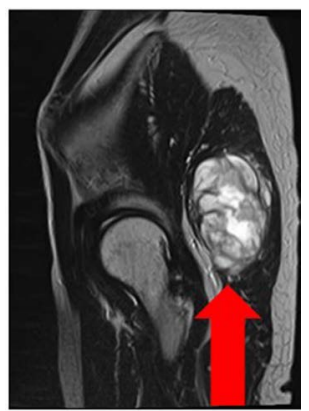

(b)

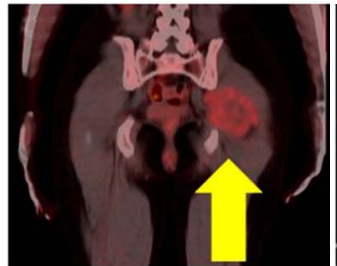

(c)

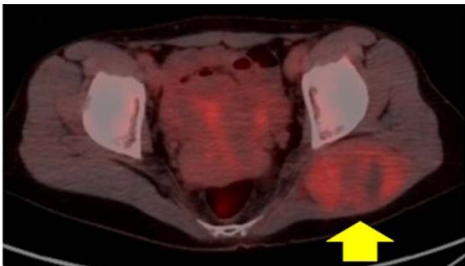

(d)

Figure 2. (a) T2-weighted coronal image of the tumor; (b) T2-weighted sagittal image of the tumor. Red arrows indicate the tumor mass; (c) 18F-fluorodeoxyglucose positron emission tomography (18FDG-PET) coronal image of the tumor; (d) 18FDG-PET sagittal image of the tumor. Yellow arrows indicate the tumor mass.

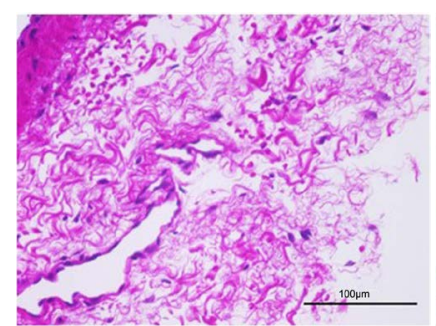

(a)

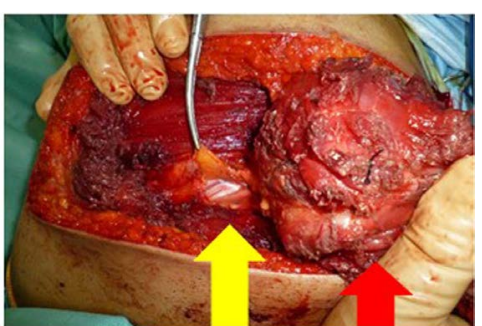

(b)

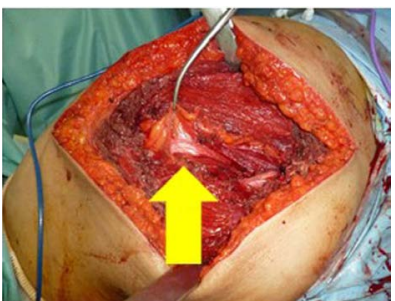

(c)

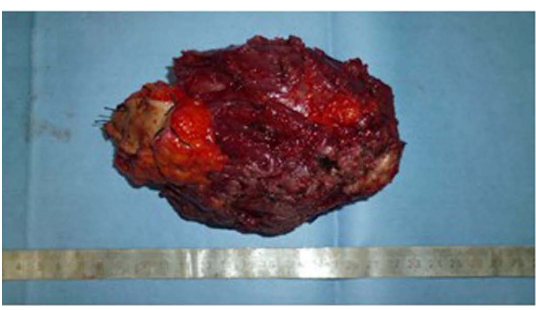

(d)

Figure 3. (a) Histology of the tumor with hematoxylin and eosin staining by needle biopsy, $\times 400$ magnification; (b) and (c) Findings at the operative site; (d) Macroscopic finding of the resected specimen. Red arrows indicate the tumor mass; yellow arrows indicate the sciatic nerve.

2/50 nuclear fissions under a high-power field (Figure 4(a) and Figure 4(b)). Immunohistochemical analysis revealed MUC4 positivity (Figure 4(c)), while no S-100- and MDM2-positive cells were observed (Figure 4(d) and Figure 4(e)). CD34-positive cells were observed in the vasculature (Figure 4(f)). The final diagnosis was a low-grade fibromyxoid sarcoma, and gluteal muscle was 


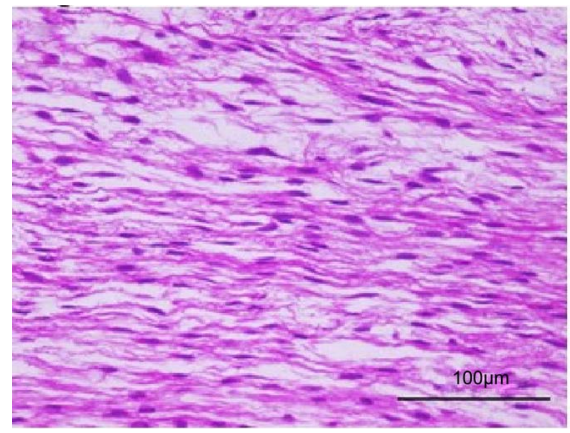

(a)

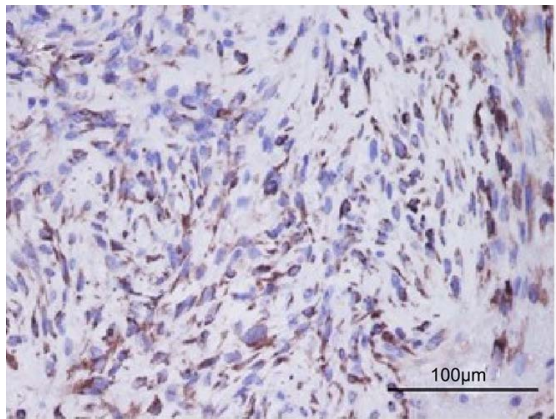

(c)

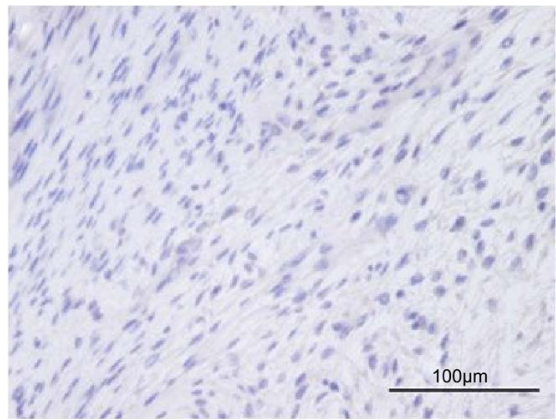

(e)

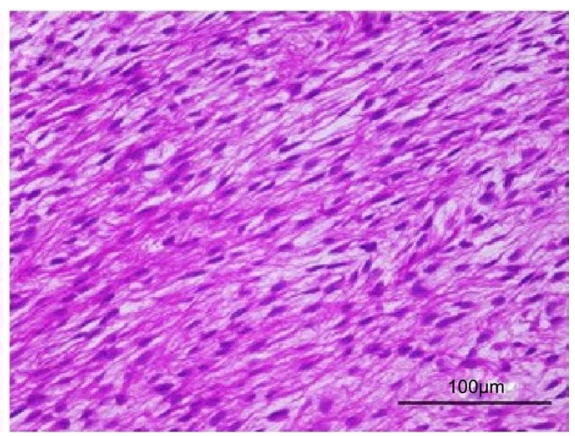

(b)

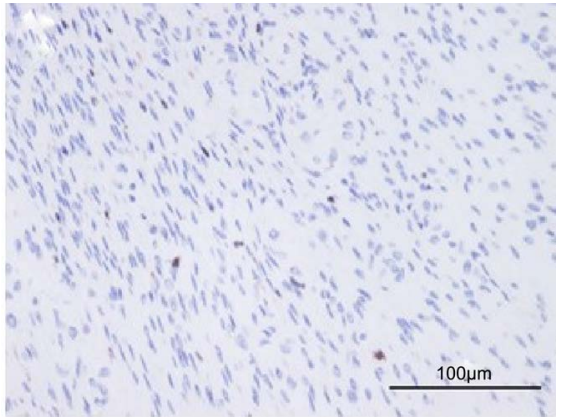

(d)

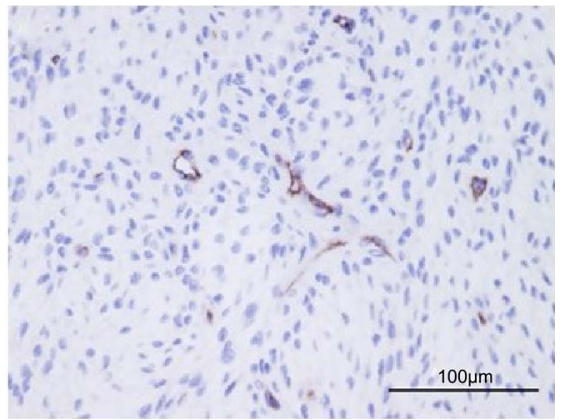

(f)

Figure 4. (a) and (b) Hematoxylin and eosin staining of the resected specimen; (c) Immunohistochemical staining for MUC4; (d) Immunohistochemical staining for S-100; (e) Immunohistochemical staining for MDM2; (f) Immunohistochemical staining for CD34. All images are $\times 400$ magnification.

present between the tumor and sciatic nerve. Her sciatic-like pain had resolved 6 months after the operation, and no recurrence or metastasis of the tumor has been observed 6 months on.

\section{Discussion}

We report what is to our knowledge the first patient with a low-grade myxofibrosarcoma in the gluteal region presenting as sciatic nerve pain. The gluteal muscle was present between the tumor and sciatic nerve; however, the mass was large and elastic-hard, and the patient's pain was exacerbated by pressure resulting from tumor compression. The peculiarities in the present case are that the tumor caused sciatic nerve pain because the mass was large and of its com- 
pression.

There are only a few previous reports of low-grade fibromyxoid sarcomas that were investigated using 18FDG-PET; the SUV max in these cases ranged from 1.4 to 4.0 [2] [3] [4] [5], which was consistent with that observed for our patient's tumor.

In general, low-grade fibromyxoid sarcoma exhibits fibrous and myxoid areas comprising spindle- and asteroid fibroblast-like tumor cells in a myxoid background as evident on hematoxylin and eosin staining [5]; this was also true in our patient. Diagnosing low-grade fibromyxoid sarcoma morphologically may be challenging owing to its typically low cellularity, abundant collagen, and bland cytomorphology. It is important to distinguish this tumor type from benign or low-grade fibromyxoid lesions because of the significant potential for recurrence and late metastatic spread [6] [7] [8]. The differential diagnosis of low-grade fibromyxoid sarcoma includes nodular fasciitis, myxoma, desmoid fibromatosis, perineurioma, neurofibroma, dermatofibrosarcoma protuberans, ossifying fibromyxoid tumor, and low-grade myxofibrosarcoma. Immunohistochemistry is useful for diagnosis, but markers are of limited values owing to the non-specificity of low-grade fibromyxoid sarcoma's staining profile. For instance, S-100, MDM2, and CD34 were negative in our patient (Figure 4) as they were in previously reported patients [9] [10]. Doyle et al. identified MUC4 as a highly sensitive and specific immunohistochemical marker for low-grade fibromyxoid sarcoma [11]; all 49 patients with low-grade fibromyxoid sarcoma in their study showed cytoplasmic staining for MUC4, while all other tumor types (other than $30 \%$ of monophasic synovial sarcomas) were negative for this protein. Among other soft tissue tumors, MUC4 is also a sensitive and useful marker for identifying sclerosing epithelioid fibrosarcoma, which has similarities to low-grade fibromyxoid sarcoma [12]. Our diagnosis of low-grade fibromyxoid sarcoma was based on all our histochemical findings taken together.

FUS gene rearrangement as detected by fluorescence in situ hybridization, as well as FUS-CREB3L2/FUS-CREB3L1 chimeric fusion genes detected by reverse transcription polymerase chain reaction, are other reliable approaches for diagnosing low-grade fibromyxoid sarcoma [13]. MUC4-negative tumors that are suspected of being low-grade fibromyxoid sarcoma should undergo FUS gene rearrangement analysis to aid the diagnosis [13]. We did not perform these analyses in our patient because her tumor was positive for MUC4.

Surgical resection remains the standard treatment for low-grade fibromyxoid sarcoma, with wide surgical resection being the most effective strategy [10] [14]. The benefit of radiotherapy remains questionable and is performed only in patients at risk for recurrence or metastasis such as margin positivity, tumor location, and tumor size [10]. Chemotherapy is reserved for patients whose tumors recur locally or spread to distant sites [14] [15]. No adjuvant treatments were administered to our patient as they would have been unconventional for low-grade fibromyxoid sarcoma. 


\section{Conclusion}

We describe a patient with low-grade fibromyxoid sarcoma in the left gluteal region presenting as sciatic nerve pain. Oncologists should consider that low-grade fibromyxoid sarcoma of the gluteal region can induce sciatic nerve pain. As the rates of recurrence and metastasis are high, long-term follow-up is necessary.

\section{Acknowledgements}

We would like to thank Editage (http://www.editage.jp) for English language editing.

\section{References}

[1] Evans, H.L. (1987) Low-Grade Fibromyxoid Sarcoma. A Report of Two Metastasizing Neoplasms Having a Deceptively Benign Appearance. American Journal of Clinical Pathology, 88, 615-619. https://doi.org/10.1093/ajcp/88.5.615

[2] Williams, H.T., Gossage Jr., J.R., Allred, T.J., Kallab, A.M., Pancholy, A. and Anstadt, M.P. (2004) F-18 FDG Positron Emission Tomography Imaging of Rare Soft Tissue Sarcomas: Low-Grade Fibromyxoid Sarcoma and Malignant Hemangiopericytoma. Clinical Nuclear Medicine, 29, 581-584.

https://doi.org/10.1097/01.rlu.0000134996.94657.e2

[3] Yamashita, H., Endo, K., Takeda, C., Teshima, R., Osaki, M. and Yoshida, H. (2013) Intramuscular Myxoma of the Buttock Mimicking Low-Grade Fibromyxoid Sarcoma: Diagnostic Usefulness of MUC4 Expression. Skeletal Radiology, 42, 1475-1479. https://doi.org/10.1007/s00256-013-1641-4

[4] Konecna, J., Liberale, G., Haddad, J., de Saint-Aubain, N. and El Nakadi, I. (2015) Diffuse Intra-Abdominal Low Grade Fibromyxoid Sarcoma with Hepatic Metastases: Case Report and Review of the Literature. International Journal of Surgery Case Reports, 14, 40-43. https://doi.org/10.1016/j.ijscr.2015.06.022

[5] Hashimoto, M., Koide, K., Arita, M., Kawaguchi, K., Mikuriya, Y., Iwata, J. and Iwamoto, T. (2016) A Low-Grade Fibromyxoid Sarcoma of the Internal Abdominal Oblique Muscle. Case Reports in Surgery, 2016, Article ID: 8524030. https://doi.org/10.1155/2016/8524030

[6] Evans, H.L. (1993) Low-Grade Fibromyxoid Sarcoma: A Report of 12 Cases. The American Journal of Surgical Pathology, 17, 595-600. https://doi.org/10.1097/00000478-199306000-00007

[7] Folpe, A.L., Lane, K.L., Paull, G. and Weiss, S.W. (2000) Low-Grade Fibromyxoid Sarcoma and Hyalinizing Spindle Cell Tumor with Giant Rosettes: A Clinicopathologic Study of 73 Cases Supporting Their Identity and Assessing the Impact of High-Grade Areas. The American Journal of Surgical Pathology, 24, 1353-1360. https://doi.org/10.1097/00000478-200010000-00004

[8] Goodlad, J.R., Mentzel, T. and Fletcher, C.D. (1995) Low Grade Fibromyxoid Sarcoma: Clinicopathological Analysis of Eleven New Cases in Support of a Distinct Entity. Histopathology, 26, 229-237. https://doi.org/10.1111/j.1365-2559.1995.tb01436.x

[9] Kurisaki-Arakawa, A., Suehara, Y., Arakawa, A., Takagi, T., Takahashi, M., Mitani, K., Kaneko, K., Yao, T. and Saito, T. (2014) Deeply Located Low-Grade Fibromyxoid Sarcoma with fus-creb312 Gene Fusion in a 5-Year-Old Boy with Review of Literature. Diagnostic Pathology, 9, 163. https://doi.org/10.1186/s13000-014-0163-2 
[10] Ban, L.K., Tseng, A.H., Huang, S.-H. and Lee, H.H. (2017) Low-Grade Fibromyxoid Sarcoma of the External Anal Sphincter: A Case Report. World Journal of Surgical Oncology, 15, 109. https://doi.org/10.1186/s12957-017-1174-8

[11] Doyle, L.A., Möller, E., Dal Cin, P., Fletcher, C.D., Mertens, F. and Hornick, J.L. (2011) MUC4 Is a Highly Sensitive and Specific Marker for Low-Grade Fibromyxoid Sarcoma. The American Journal of Surgical Pathology, 35, 733-741. https://doi.org/10.1097/PAS.0b013e318210c268

[12] Doyle, L.A., Wang, W.-L., Dal Cin, P., Lopez-Terrada, D., Mertens, F., Lazar, A.J., Fletcher, C.D. and Honick, J.L. (2012) MUC4 Is a Sensitive and Extremely Useful Marker for Sclerosing Epithelioid Fibrosarcoma: Association with FUS Gene Rearrangement. The American Journal of Surgical Pathology, 36, 1444-1451. https://doi.org/10.1097/PAS.0b013e3182562bf8

[13] Patel, R.M., Downs-Kelly, E., Dandekar, M.N., Fanburg-Smith, J.C., Billings, S.D., Tubbs, R.R. and Goldblum, J.R. (2011) Fus (16p11) Gene Rearrangement as Detected by Fluorescence In-Situ Hybridization in Cutaneous Low-Grade Fibromyxoid Sarcoma: A Potential Diagnostic Tool. The American Journal of Dermatopathology, 33, 140-143. https://doi.org/10.1097/IAE.0b013e318176de80

[14] Tang, Z., Zhou, Z.-H., Lv, C.-T., Qin, L.Y., Wang, Y., Tian, G., Luo, X.-L., Zhu, Q. and Xu, X.-G. (2010) Low-Grade Fibromyxoid Sarcoma: Clinical Study and Case Report. Journal of Oral and Maxillofacial Surgery, 68, 873-884. https://doi.org/10.1097/IAE.0b013e318176de80

[15] Maretty-Nielsen, K., Baerentzen, S., Keller, J., Dyrop, H.B. and Safwat, A. (2013) Low-Grade Fibromyxoid Sarcoma: Incidence, Treatment Strategy of Metastases, and Clinical Significance of the FUS Gene. Sarcoma, 2013, Article ID: 256280. https://doi.org/10.1155/2013/256280 